\title{
Improving hormonal therapy: to extend, to intermit, or to readjust expectations?
}

\author{
Iris Noordhoek, Esther Bastiaannet, Cornelis J. H. van de Velde \\ Department of Surgery, Leiden University Medical Center, Leiden, The Netherlands \\ Correspondence to: Prof. Dr. Cornelis J. H. van de Velde. Department of Surgery, Leiden University Medical Center, Albinusdreef 2, 2300 RC Leiden, \\ The Netherlands. Email: c.j.h.van_de_velde@lumc.nl. \\ Comment on: Colleoni M, Luo W, Karlsson P, et al. Extended adjuvant intermittent letrozole versus continuous letrozole in postmenopausal women \\ with breast cancer (SOLE): a multicentre, open-label, randomised, phase 3 trial. Lancet Oncol 2018;19:127-38.
}

Submitted Mar 15, 2018. Accepted for publication Mar 28, 2018.

doi: $10.21037 /$ tcr.2018.04.01

View this article at: http://dx.doi.org/10.21037/tcr.2018.04.01

For quite some time now, a discussion has been going on in the world of breast cancer research concerning the optimal duration of hormonal therapy (HT) in hormone receptor (HR) positive early breast cancer. Multiple trials have tried providing closure to this discussion, but so far, none have succeeded (1).

What we do know, is that when patients are treated with merely tamoxifen for 5 years, disease free survival (DFS) can be improved by treating these patients with another 5 years of tamoxifen or another 2.5 years with an aromatase inhibitor (AI), alternatively. In contrast, overall survival (OS) is not improved when extending therapy beyond the initial 5 years (2-4). It is still unclear whether extending treatment improves DFS or OS when patients are treated with an AI in the initial 5 years, though not for lack of trying. For example, in the MA.17R trial (5), patients received 3 to 5 years of tamoxifen, followed by either 10 years of letrozole or 5 years of letrozole and 5 years of placebo. A small, significant difference was found for 5 -year DFS; $95 \%$ and $91 \%$, respectively, hazard ratio (HR) 0.66 with a $95 \%$ confidence interval (CI) of 0.48 to 0.91 . Though, no difference was found for OS. Also, the trial did not included analysis for minimal clinically important differences (MCID), therefore, the clinical relevance of these statistical differences can be debated $(6,7)$. In the NSABP B-42 trial (8), patients were initially treated with 5 years of AI or 2-3 years of tamoxifen followed by 2-3 years of AI, and were then randomized to receive extended treatment for 5 years with letrozole or a placebo. No differences were found in DFS or OS. The IDEAL trial (9) compared extending therapy with letrozole for 2.5 years or 5 years after an initial 5 years treatment with tamoxifen or AI. Again, no differences were found in DFS or OS. As evaluated by Helwick (6), the B-42 and IDEAL trial seem methodologically sound and the results are deemed comparable.

Another recently published trial, the study of letrozole extension (SOLE) trial by the International Breast Cancer Study Group (IBCSG) (10), seeks to uncover new insights regarding HT. Instead of studying whether HT should be extended, the study focusses on different methods of administering extended HT; continuously or intermittently. It is a phase 3 clinical trial that randomized postmenopausal women initially treated with 4 to 6 years of HT (tamoxifen, AI or a combination) to continuous letrozole for 5 years or 9 months on and 3 months off letrozole for 4 years and 12 months on letrozole for the 5 th year. The study unfortunately did not include a treatment arm in which therapy was not extended beyond the initial 5 years. Nevertheless, the concept of administering AI intermittently as opposed to continuously is an interesting idea. It is based on several preclinical trials (11-14) demonstrating that when HR positive tumor cells have been deprived of estrogen for a long period of time, sudden exposure to high doses of estrogen can induce cell death.

However, when translating this concept to the study design of the SOLE trial, the IBCSG did not incorporate an important consideration. Merely discontinuing letrozole for 3 months is probably not sufficient to yield adequate amounts of estrogen to induce cell death. The effect of letrozole will take about 2 weeks to completely wear off, and the remaining time is not enough for the women to produce the amounts of estrogen necessary $(15,16)$. As mentioned in the commentary by Chlebowski and Pan (17), 
to reflect the preclinical study designs, the patients should have received additional estrogen in the period off letrozole to appropriately study the cytocidal effect of estrogen.

Another curiosity in this study, as also pointed out by Chlebowski et al. (17), is the definition of the inclusion criterium "postmenopausal". It was defined as women of any age either having had a bilateral oophorectomy or radiation castration and amenorrhea for more than 3 months, or women of any age having biochemical evidence of postmenopausal status, based on serum levels of estradiol, follicle-stimulating hormone and luteinizing hormone. Yet, patients previously having received tamoxifen were also eligible for inclusion. Tamoxifen is known to mimic (biochemical) postmenopausal status, and some of these patients resume menses after stopping with tamoxifen (18). Patients who are not truly postmenopausal will have no benefit of letrozole (18), whether in the intermittent or the continuous arm. Including these patients will not necessarily create differences between the two arms, but will obscure some of the treatment effect. Moreover, even if it can be confirmed that all included patients who had undergone previous treatment with tamoxifen were truly postmenopausal, they will have had a shorter period of estrogen deprivation than patients previously treated with AI (9 months versus 4-6 years). Since this issue does not arise in the continuous arm, the observed difference in treatment effect between the groups might thus be influenced by including tamoxifen-treated patients. It should also be noted that $11-16 \%$ of the patients in the experimental arm did not stop letrozole according to protocol, which can contribute to concealment of any treatment effect, as well.

The primary endpoint was DFS, defined as time from randomization to the occurrence of local, regional or distant relapse of breast cancer, a second cancer, or death without second cancer or recurrence; the results showed no difference in 5 -year DFS between the intermittent and continuous group (85.8\% and $87.5 \%$, respectively, HR 1.08 , 95\% CI, 0.93-1.26). As the authors already pointed out in the discussion section, this classical definition including both breast cancer related and unrelated events, might have obscured differences between the groups, as many breast cancer unrelated events, such as second non-breast malignancy occurred in both groups. Be that as it may, one of the secondary outcome measures that were studied, was breast cancer free interval (BCFI) and this was not statistically significantly different between the two groups either. Other secondary outcome measures that were studied included OS and occurrence of adverse events (AE), none of which were significantly different between both groups.
What can be concluded from this, is that it might be beneficial to administer letrozole intermittently as opposed to continuously, but the SOLE trial is not designed to demonstrate this, due to its methodological flaws (19). The authors conclude that, at the least, intermittently is a safe way of administering letrozole and yields comparable results to continuous administration. As the follow-up of this trial is only 5 years, these deductions are a bit too blunt and longer follow-up is needed to assume safety.

Lastly, the study included a quality of life (QoL) analysis, as well. This was measured in a subset of patients, comprising $20 \%$ of the original participants. This subset differed significantly from the overall group in terms of number of patients who were premenopausal at diagnosis and were previously treated with tamoxifen, instead of AI of a combination. The reason for choosing to perform the QoL analysis in a smaller group of patients is not described in the article. Therefore, any results cannot be projected onto the overall study population. At 12 months, small but statistically significant differences in QoL were seen in favor of the intermittent group, however, at 24 months, these differences were no longer significant. Thus, none of the studied outcome parameters showed statistically significant differences between treatment with intermittent or continuous letrozole.

The regrettable characteristic that all these trials studying extended HT have in common, is the lack of translating statistical significance (when present) to clinical relevance. Any significant differences must be proportionate to the considerable side effects that occur when using an AI. Furthermore, the idea of extending HT is based on initial treatment with tamoxifen. However, AIs have proved to be superior to tamoxifen and current guidelines for treating postmenopausal patients with HR positive breast cancer recommend treatment with an AI. Since there has been no evidence as of yet to support extending treatment after initial treatment with an $\mathrm{AI}$, maybe it is time to readjust our expectations and to accept 5 years of treatment with an AI as sufficient.

\section{Acknowledgments}

Funding: None.

\section{Footnote}

Provenance and Peer Review: This article was commissioned and reviewed by the Section Editor San-Gang Wu (Department of Radiation Oncology, Xiamen Cancer Center, The First Affiliated Hospital of Xiamen University, Xiamen, China). 
Conflicts of Interest: All authors have completed the ICMJE uniform disclosure form (available at http://dx.doi. org/10.21037/tcr.2018.04.01). The authors have no conflicts of interest to declare.

Ethical Statement: The authors are accountable for all aspects of the work in ensuring that questions related to the accuracy or integrity of any part of the work are appropriately investigated and resolved.

Open Access Statement: This is an Open Access article distributed in accordance with the Creative Commons Attribution-NonCommercial-NoDerivs 4.0 International License (CC BY-NC-ND 4.0), which permits the noncommercial replication and distribution of the article with the strict proviso that no changes or edits are made and the original work is properly cited (including links to both the formal publication through the relevant DOI and the license). See: https://creativecommons.org/licenses/by-nc-nd/4.0/.

\section{References}

1. Wimmer K, Strobl S, Bolliger M, et al. Optimal duration of adjuvant endocrine therapy: how to apply the newest data. Ther Adv Med Oncol 2017;9:679-92.

2. Al-Mubarak M, Tibau A, Templeton AJ, et al. Extended adjuvant tamoxifen for early breast cancer: a meta-analysis. PLoS One 2014;9:e88238.

3. Davies C, Pan H, Godwin J, et al. Long-term effects of continuing adjuvant tamoxifen to 10 years versus stopping at 5 years after diagnosis of oestrogen receptorpositive breast cancer: ATLAS, a randomised trial. Lancet 2013;381:805-16.

4. Goss PE, Ingle JN, Martino S, et al. A Randomized Trial of Letrozole in Postmenopausal Women after Five Years of Tamoxifen Therapy for Early-Stage Breast Cancer. New England Journal of Medicine 2003;349:1793-802.

5. Goss PE, Ingle JN, Pritchard KI, et al. Extending Aromatase-Inhibitor Adjuvant Therapy to 10 Years. The New England journal of medicine 2016;375:209-19.

6. Helwick C. Benefit of Extended Adjuvant Endocrine Therapy Not Upheld in Multiple Studies Reported in San Antonio. The ASCO Post 2016.

7. Cook CE. Clinimetrics Corner: The Minimal Clinically Important Change Score (MCID): A Necessary Pretense. J Man Manip Ther 2008;16:E82-3.

8. Mamounas EP, Lembersky B, Jeong JH, et al. NSABP B-42: a clinical trial to determine the efficacy of five years of letrozole compared with placebo in patients completing five years of hormonal therapy consisting of an aromatase inhibitor (AI) or tamoxifen followed by an $\mathrm{AI}$ in prolonging disease-free survival in postmenopausal women with hormone receptor-positive breast cancer. Clin Breast Cancer 2006;7:416-21.

9. Blok EJ, Kroep JR, Meershoek-Klein Kranenbarg E, et al. Optimal Duration of Extended Adjuvant Endocrine Therapy for Early Breast Cancer; Results of the IDEAL Trial (BOOG 2006-05). J Natl Cancer Inst 2018;110.

10. Colleoni M, Luo W, Karlsson P, et al. Extended adjuvant intermittent letrozole versus continuous letrozole in postmenopausal women with breast cancer (SOLE): a multicentre, open-label, randomised, phase 3 trial. The Lancet Oncology 2018;19:127-38.

11. Lewis JS, Osipo C, Meeke K, et al. Estrogen-induced apoptosis in a breast cancer model resistant to longterm estrogen withdrawal. J Steroid Biochem Mol Biol 2005;94:131-41.

12. Sabnis GJ, Macedo LF, Goloubeva O, et al. Stopping treatment can reverse acquired resistance to letrozole. Cancer Res 2008;68:4518-24.

13. Song RX, Mor G, Naftolin F, et al. Effect of long-term estrogen deprivation on apoptotic responses of breast cancer cells to 17beta-estradiol. J Natl Cancer Inst 2001;93:1714-23.

14. Yao K, Lee ES, Bentrem DJ, et al. Antitumor action of physiological estradiol on tamoxifen-stimulated breast tumors grown in athymic mice. Clin Cancer Res 2000;6:2028-36.

15. Anderson GL, Chlebowski RT, Aragaki AK, et al. Conjugated equine oestrogen and breast cancer incidence and mortality in postmenopausal women with hysterectomy: extended follow-up of the Women's Health Initiative randomised placebo-controlled trial. Lancet Oncol 2012;13:476-86.

16. Ellis MJ, Gao F, Dehdashti F, et al. Lower-dose vs highdose oral estradiol therapy of hormone receptor-positive, aromatase inhibitor-resistant advanced breast cancer: a phase 2 randomized study. Jama 2009;302:774-80.

17. Chlebowski RT, Pan K. Complexity of intermittent letrozole adjuvant therapy. Lancet Oncol 2018;19:13-5.

18. Smith IE, Dowsett M, Yap YS, et al. Adjuvant aromatase inhibitors for early breast cancer after chemotherapyinduced amenorrhoea: caution and suggested guidelines. J Clin Oncol 2006;24:2444-7.

19. Jordan VC. The Study of Letrozole Extension (SOLE) revisited. Lancet Oncol 2018;19:e77.

Cite this article as: Noordhoek I, Bastiaannet E, van de Velde CJ. Improving hormonal therapy: to extend, to intermit, or to readjust expectations? Transl Cancer Res 2018;7(Suppl 4):S525S527. doi: 10.21037/tcr.2018.04.01 